\title{
Adult Intussusceptions: A Series of 5 Cases
}

\author{
Dr. Pranab Kumar Ghosh ${ }^{1}$, Dr. Goutam Dhar ${ }^{4}$, Dr. Ashis Kumar Saha ${ }^{1}$, \\ Dr Goutam Ghosh ${ }^{2}$, Dr Nilay Mandal ${ }^{1}$, Dr. Chhanda Das ${ }^{3}$, Dr. Bikramjit Maity ${ }^{1}$ \\ ${ }^{I}$ Department of Surgery, Bankura Sammilani Medical College, Bankura, India \\ ${ }^{2}$ Department of Surgery, R. G. Kar Medical College, Kolkata, India \\ ${ }^{3}$ Department of Pathology, R. G. Kar Medical College, Kolkata, India \\ ${ }^{4}$ Department of Community Medicine, N. R. S. Medical College, Kolkata, India)
}

\begin{abstract}
Intussusception in adult patients is rare in comparison to paediatric patients. We are hereby reporting a series of five cases of adult intussusception. In most of the cases, USG findings were diagnostic and organic lesions were found as causative factors but the diagnosis of intussusceptions from any cause in pregnancy is made more difficult by the common overlapping complaints of nausea, vomiting and abdominal pain, which may persist during the second trimester. The very low incidence rate of 1/30,000 of all hospital admissions makes it far more difficult to diagnose. A very much vigilant mind can only reduce the morbidity out of this condition by use of meticulous Ultrasonography
\end{abstract}

Key wards: intussusception, Ileo-caecal, colo-colic,

\section{Introduction:}

Intussusception, the invagination of proximal intestinal segment into the distal segment is a common cause of pediatric intestinal obstruction. Intussusception in adult population is rare; accounting for only $5 \%$ of all intussusceptions $[1,2,3,4]$ and particularly so in pregnant women ( $1 / 68,000$ deliveries)[4]. In contrast to the pediatric population, where intussusception is usually idiopathic or due to viral illness, an organic lesion is usually identifiable as the cause of adult intussusceptions in about $90 \%$ of cases [3] and frequently a tumor [4] The diagnosis of intussusceptions from any cause in pregnancy is made more difficult by the common overlapping complaints of nausea, vomiting and abdominal pain, which may persist during the second trimester [2,5] and (also relative contraindication of x-ray and CT scan). It is a potentially life-threatening condition for both the mother and the fetus [4,2] challenging both gynaecologists and surgeon as diagnosis is difficult and emergency surgery indicated[2].

Case 1:

\section{Case Series:}

A 60yr old male patient presented with left lower abdominal pain, vomiting and fever for last 3 days. There was no H/O loose stool or bleeding per rectum. On examination, abdomen soft, IPS present; an ill defined tender intraperitoneal mass was palpable at lower abdomen left to the umbillicus. On rectal examination, no ballooning or growth was palpable. Ultrasonography showed 'gut in gut' appearance suggestive of intussusception. On exploration Ileo-Ileal intussusception noted, which was reduced by manual reduction. After reduction it was found that about $12 \mathrm{~cm}$ of ileum was gangrenous, $15 \mathrm{~cm}$ proximal to Ileo-caecal junction, no lead point was found. Resection of the gangrenous part followed by end-end anastomosis was done. The pt had uneventful recovery and was discharged on 11 th post-op period.

Case 2:

A $65 \mathrm{yr}$ old male patient with past $\mathrm{H} / \mathrm{O}$ ischaemic heart disease presented with bleeding per rectum and lower abdominal pain for last 5 days associated with vomiting. On clinical examination, it was found that patient was dehydrated ,pulse 110/min, BP -180/110 mm Hg. Per abdominal examination revealed a soft, non-tender, intraperitoneal lump in left lumber region with ill defined margins. Classical redcurrant jelly like stool on gloved fingertip after digital rectal examination.USG showed hepatomegaly, bilateral echogenic kidney, gallbladder containing sludge. CECT abdomen revealed colo-colic intussusception with a polypoidal growth as a lead point. On exploration, colo-colic intussusceptions noted, a pedunculated polyp was found as lead point. Resection of involved sigmoid colon along with the polyp was done. Patient was kept in ITU but gradually developed septicaemia, progressive renal failure and expired on 3rd postoperative day. Histopathological examination of resected polyp revealed it as intestinal lipoma. 
Case 3:

A $63 \mathrm{yr}$ old male patient was admitted with pain in right side of abdomen, but no history of vomiting, fever or bleeding per rectum. Vitals were stable. On examination, abdomen was soft, non-tender, no palpable lump.USG and straight x-ray abdomen revealed no abnormality. Patient was put on conservative management, but did not respond satisfactorily. Repeat USG and straight x-ray abdomen was inclusive, so CT scan was planned. CT scan report revealed ileocolic intussusception with a polypoidal lesion as a lead point. On exploration, ileocolic intussusception was noted with a pedunculated polyp as a lead point associated with gangrenous changes in the intussusceptum. Right hemicaolectomy was done. Histopathological report of the pedunculated polyp showed it was intestinal lipoma.

Case 4:

A 45yr male patient presented with pain in left iliac fossa, vomiting and passage of mucous mixed bloody stool for last 2 days. On examination, an ill defined lump was palpable in left iliac fossa.USG showed features suggestive of intussusception. On exploration, ileo-ileal intussusception noted. After manual reduction, enlarged lymph nodes at mesenteric border were seen as the leadpoint. Enlarged Lymph nodes were sent for histopathology. Histopathology report suggested tuberculous lyphadenopathy. Patient was started on antitubercular drugs.

\section{Case 5:}

A $22 \mathrm{yr}$ old, 16 wks pregnant mother was admitted with acute onset severe cramp like upper abdominal pain and non-bilious vomiting for last 2 days. The pain was increasing on jolting and walking. On abdominal examination, tender point was present at right supra-umbilical region, but no rebound tenderness, IPS was present. Initial suspicion was acute appendicitis. USG revealed 16 wks live foetus with conglomerated gut loops suggestive of sub acute intestinal obstruction, appendix was normal. Pt was put on conservative management. Next day, there was no further vomiting but no improvement in abdominal pain and tenderness. Review USG done on next morning revealed 'gut in gut' appearance suggestive of intussusception at sub-hepatic region with length of intussusceptum about $3.5 \mathrm{~cm}$ After consultation with gynaecologist, exploratory laparotomy was done, ileo-ileal intussusception at sub-hepatic region was noted. On manual reduction inverted Meckel's diverticulum as leadpoint along with gangrenous changes in surrounding small bowel was noted. Approximately $20 \mathrm{~cm}$ of small intestine along with the Meckel's Diverticulum was resected followed by end to end anastomosis. The patient had inevitable abortion on 7th post-op day; otherwise the mother had uneventful recovery from surgical point of view. She was discharged on 11th post-op day.

\section{Discussions:}

Intussusceptions were usually fatal till early 20th century. John Hunter described the clinic pathological characteristics. Sir Fredrick Treves, an eminent 19th century surgeon described the plan of treatment, which by and large remains valid to date [6].

Intussusceptions are most common in childhood accounting for $15 \%$ of bowel obstructions. Intussusceptions in adults accounts for only $5 \%$ of the total number of intussusceptions $[1,2,3,4,6]$.

Intussusceptions is expected to be found in 1/30,000 of all hospital admissions, 1/1300 of all abdominal operations, $1 / 30-1 / 100$ of all cases operated for intestinal obstruction[2,6]. The mean age at presentation tends to be in the 6th decade of life between 51 - 54.4 years [2,6]. It may be acute or chronic (persistent or intermittent) in addition to being 'silent'. The chronic intussusceptions may have lasted in some instances for a year before the diagnosis.

The male to female ratio is $1: 1-1.3(3.6: 1$ for children)[2,6]. The age range is from intrauterine life to the 9 th decade, the mean being 54.4years for adults and 2.2years for children [6].

The clinical presentation in adult intussusception is often chronic, and most patients present with nonspecific symptoms that are suggestive of intestinal obstruction [7]. The classical triad of pain abdomen, abdominal lump and red currant jelly is rarely encountered in cases of adult intussusception [1]. Wang et al[8], in their review of 41 cases of adult intussusception, reported that only $9.8 \%$ of patients had this classical triad. Abdominal pain is the most common symptom (71-90\%) followed by vomiting and bleeding per rectum [1]. Adult intussusception is most commonly seen in small intestine [6]. About $90 \%$ of occurrences in adults have a lead point, a well-definable pathological abnormality. In general, the majority of lead points in the small intestine consist of benign lesions, malignant lesions causing intussusception in small bowel are usually metastatic in nature, malignant melanoma being the most common [1]. Malignant lesions (either primary or metastatic) account for up to $30 \%$ of cases of intussusception in the small intestine. On the other hand, intussusception occurring in the large bowel is more likely to have a malignant etiology and represents up to $66 \%$ of the cases [6,7]. Idiopathic intussusception occurs in only $6.6-15 \%$ of the adult cases as compared to the children when it may be as much as $41 \%$ [6]. 
Abdominal CECT is the most accurate imaging modality for intussusceptions followed by ultrasound $[1,2,7]$. Towards the diagnosis, often being hitherto unsuspected, the CT/MRI may have a diagnostic yield of $52 \%$, as compared to $41 \%$ for contrast GI studies 33 and $32 \%$ for the ultrasound. The classical CT/MR finding is the 'target lesion' formed by bowel-within-bowel, a 'double ring' or a 'coiled spring' appearance. Others are presence of an intra-luminal soft tissue mass and an eccentric mesentry [6].

Ultrasound of abdomen is very useful in a developing country like India, where emergency CECT facility is not universally available. Ultrasonography may not be a satisfying investigation in acute presentation as findings are difficult to appreciate in view of gas filled bowel loops. Classical sonographic features include the "target" or "doughnut" signs on the transverse view and the "pseudo-kidney" sign or the "hay-fork" sign in the longitudinal view [1,7].
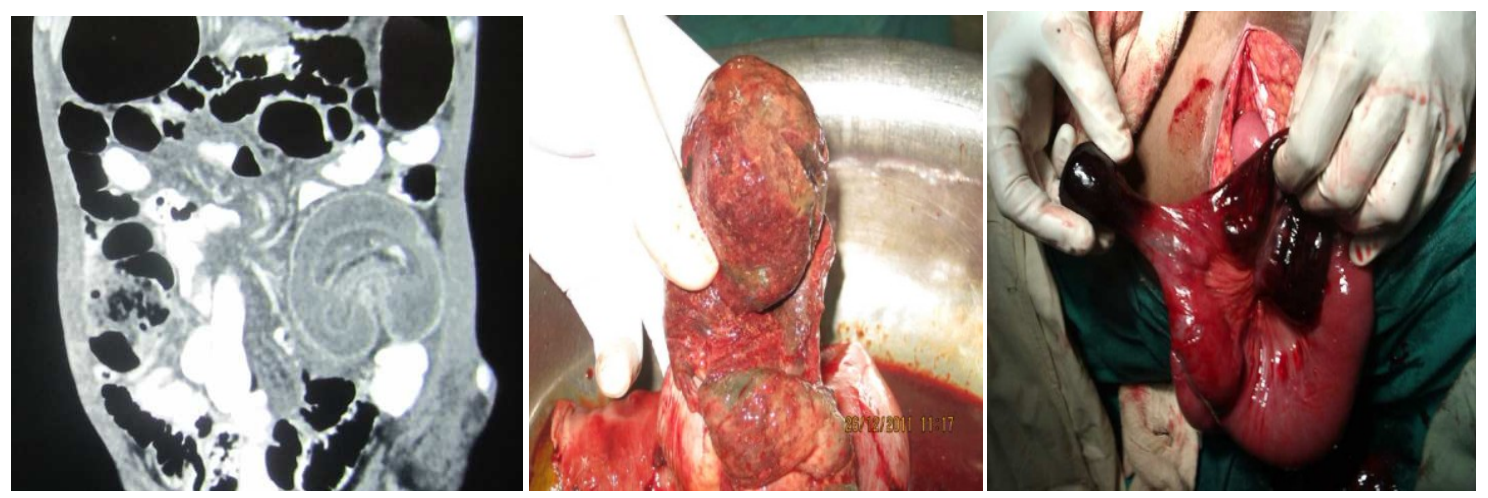

The treatment of intussusception in the adult is almost always surgery in view of large proportion of underlying abnormalities and associated neoplasm, associated gangrenous changes [1,2,6]. Dharmendra Prasad et all reported that $77 \%$ patients had associated bowel gangrene [1].

Intussusception is difficult to diagnose at the best of times in adults but in pregnancy even more so. Some of the presenting symptoms like abdominal pain and vomiting are common symptoms in pregnancy. Furthermore, it is not only the life of the mother that is at risk but also the life of the foetus [2,4,5]. Intussusception in pregnancy is rare. Intestinal obstruction in pregnancy has been reported to occur with an incidence between 1:2500-3500, most commonly secondary to adhesions or gastrointestinal volvulus. Intestinal obstruction in pregnancy is associated with a high maternal and perinatal mortality of $6 \%$ and $26 \%$, respectively. Additionally, diagnostic imaging in pregnancy is restricted to USS and MRI, with MRI not readily available in many hospitals. Recent publications have shown ultrasound scan to be a useful imaging technique in intussusception in pregnancy. It can exclude many of the differential diagnoses such as a twisted ovarian cyst and does not carry the risk of radiation to the foetus [2].

\section{Conclusion}

The classic presentations of intussusception are not so common in adults. So the preoperative diagnosis is more difficult in adults particularly if associated with pregnancy, because other investigations (eg. CT scan) except USG is contraindicated.

References

[1]. Dharmendra Prasad et all: Adult intussusception: Is associated bowel gangrene common? Tropical Gastroenterology 2011;32(1):45-49

[2]. Andreas Luhmann et all : Intussusception in a 20 weeks pregnant woman: a case report ; Cases Journal 2009, 2:6546 doi: $10.4076 / 1757-1626-2-6546$

[3]. Edwin C. Ouyang et all: Ileocolonic Intussusception; MedGenMed. 2005; 7(3): 15. PMCID: PMC16816274.

[4]. Guyomard A et al: Spontaneous acute intussusception in a pregnant woman; J Gynecol Obstet Biol Reprod (Paris). 2010 May;39(3):251-3. doi: 10.1016/j.jgyn.2010.02.002. Epub 2010 Mar 12

[5]. Shenhav S et all :Preoperative diagnosis of intestinal intussusception in pregnancy. A case report; J Reprod Med. 2000 Jun;45(6):501-3.

[6]. Rathore MA et all: ADULT INTUSSUSCEPTION - A SURGICAL DILEMMA; J Ayub Med Coll Abbottabad 2006;18(3)

[7]. Savas Yakan et all: Intussusception in adults: Clinical characteristics, diagnosis and operative strategies ;World J Gastroenterol 2009 April 28; 15(16): 1985-1989

[8]. Wang N, Cui XY, Liu Y,.Long J, Xu YH, Guo RX, et al: Adult intussusception: A retrospective review of 41 cases; World J Gastroenterol. 2009;15:3303-81 